\title{
The phenomenon for the wood creep in the reinforced glued wooden structures
}

\author{
Svetlana Roshchina ${ }^{1}$, Mikhail Lukin ${ }^{1 *}$, Mikhail Lisyatnikov ${ }^{1}$ and Artem Koscheev ${ }^{1}$ \\ ${ }^{1}$ Vladimir State University named after Alexander Grigorievich and Nikolai Grigorievich Stoletovs, \\ Institute of Architecture Construction and Energy engineering, Gorky Street 87, Vladimir, 600000.
}

\begin{abstract}
For the composite structures based on wood, that include reinforced wooden structures, allowance for the influence of wood specific features as a building material allows us to evaluate its structural design as accurately as possible and ensure the required reliability and durability. Creep that causes wood deformations evolving in time can be referred to the specific properties of wood to a greater extent affecting the operation of the reinforced wooden structures. Taking into account the state of the issue to determine the effect of the long-acting loads on the stress-strain state of the reinforced glued wooden structures, the article considers the application of the theory of elastic heredity as the most applicable to assess the deformability of wooden structures in time. The conducted experimental and theoretical investigations and the reinforced glued wooden structures observations during the operational process have shown that their stressstrain state changes significantly under the load over time. Glue compositions used to connect reinforcement with wood ensure their reliable joint operation, however, as the result of the wood creep where wood is the basic material, the redistribution of forces between the reinforcement and wood occurs there on and off. At the same time normal stresses in the reinforcement and the glue joint "reinforcement - wood" increase in the calculated cross sections while they decrease in the wood, which is caused by a change (decrease) in the wood elastic modulus. Allowance for the wood creep in the design of the reinforced wooden structures will allow to determine the stress - strain state of the structure in the design more precisely. Therefore, when developing the reinforced wooden structures design standards, this factor must be taken into account.
\end{abstract}

\section{Introduction}

Use of the reinforcement allows to solve the node connections and structural joints of wooden structures in a new way and more efficiently that increases their prefabricability, facilitates transportation and structure erection as well as the issues of repair and reconstruction of the operating enterprises. Investigations of the reinforced wooden structures and study of their stress-strain state under short-term and long-term loads required the development of methods for calculating such structures with allowances made for the features of the structures stress-

\footnotetext{
*Corresponding author: lukin_mihail_22@mail.ru
} 
strain state, the heterogeneousness of materials [1] in the composite structure, creep of wood and glue etc [2]. The first works in this area were performed by V.Yu. Shuko, S.A. Shuko, A.Ya. Kozulin, I.M. Linkov, V.M. Sorotokin, L.S. Chebotareva, V.D. Lee, E.A. Smirnov, V.B. Kasatkin, B.V. Nakashidze and others, which were held in the RDA named after Kucherenko in Irkutsk, Vladimir, Tomsk, Khabarovsk Polytechnic Institutes, Belarusian, Novosibirsk Engineering and Construction Institutes, Institute of Automation and Control of the State Construction Committee of Belarus, Central Research Institute of Industrial Buildings and other organizations [3, 4].

Calculation of the reinforced wooden structures has been carried out according to the given geometrical characteristics of the section as well as with allowances made for the elastic-plastic work of wood on the basis of the Belyankin-Prager diagram [5]. In which the plane-sections hypothesis, compatibility of the steel-wood adhesive bonding at all operational stages of the structural behaviour [6] have been specified. However, the conducted experimental investigations have shown that the amount of the theoretical failing moment owing to the fact that wood is an anisotropic, a material with various elastic modulus, is much higher than the experimental value [7]. The calculation method for the above characteristics is widely used in the design of bending and compression-bending reinforced wooden structures operating in the elastic stage [8], however, considering the operation of the reinforced wooden structures in the elastic-plastic stage, this method requires explicitation and improvement. Despite the fact that quite a lot of investigations of theoretical and practical importance have been made in the area of calculation of reinforced wooden structures, the calculations of bending and compression- bending elements considering the long duration of the load, taking into account the factors of redistribution of strain in the component sections, cause certain difficulties since they do not take into account the actual behavior of wood, as the main material of the structures, under long-term load, deformation capacity of the connection of reinforcement with wood in the anchorage zone. A common disadvantage of the considered calculation methods is the disregard of the effect of the longacting loads and the rheological properties of wood. When calculating wooden structures it is very important to take into account the time factor determining the period of loading [9]. Works of I.I. Goldenblata, F.P. Belyankina, V.F. Yatsenko, V.M. Kochenova, Yu.M. Ivanova, V.G. Lennova, M.A. Kleiman and other authors revealed a significant effect of the long-term load on the strength and deformability of wooden elements.

One of the directions for the calculation of engineering structures is the material consideration, its effect on the load bearing capacity, stress-strain state and deformability of structures [10].

For the composite structures based on wood, that include reinforced wooden structures [11], allowance for the influence of wood specific features as a building material allows us to evaluate its structural design as accurately as possible and ensure the required reliability and durability.

Creep that causes wood deformations evolving in time can be referred to the specific properties of wood to a greater extent affecting the operation of the reinforced wooden structures. Steel reinforcing, resisting the spreading of these deformations, is the source of the emerging additional strain leading to a change in the stress-strain state.

In view of this, modern design rules require structural calculations with allowances made for the creep influence that makes it necessary to master the theory and practice of structural calculations of the reinforced wooden structures taking into account the influence of lengthy processes.

\section{Experimental investigation}


To solve the problem of determining the stress-strain state of the reinforced wooden elements, taking into account the period of loading in normal operation, we introduce the presumption that steel reinforcement works in time elastically and is rigidly connected to the wood, that is

$\varepsilon_{s}=\varepsilon_{w}-$ (where $\varepsilon_{s}-$ is the deformations in the reinforcement, $\varepsilon_{w}-$ is the deformations in the wood); the change of strain along the cross sectional height of the element in time is proportional to the distance to the neutral layer, therefore, the plane-sections hypothesis is valid.

To the present day, there is no complete physical and chemical explanation for the nature of wood creep. The most common is the point of view on the creep nature of that boils down to the fact that the development of these deformations is a consequence of the movement (extrusion) of water in the cell walls, viscous deformations and deformations of the cellfibrous skeleton.

Creep theories imply the establishment of the relationships between stresses, strains and time that can be represented as a functional relation:

$$
\varepsilon(t)=\phi[\sigma(t) ; t \text { и } \tau]
$$

where $\varepsilon(t)$ - is the total relative deformation at time $t ; \sigma(t)$ - is the strain at any time; $t$ - is the reference time; $\tau$ - is the current time coordinate.

In present-day conditions, all existing creep theories are of the phenomenological nature, that is they are based on the description of the most investigated experimental phenomena [12]. Taking into account the state of the issue to determine the effect of the long-acting loads on the stress-strain state of the reinforced glued wooden structures [13], the article considers the application of the theory of elastic heredity as the most applicable to assess the deformability of wooden structures in time.

\section{Results and Discussion}

To account for creep in the straight-line theory of elastic heredity, the relationship between stresses and the deformations of an element the Boltzmann- Volterra integral equation has been used:

$$
\varepsilon(t)=\frac{\sigma(t)}{E}+\frac{1}{E} \int_{\tau}^{t} K(t-\tau) \sigma(\tau) d \tau \phi
$$

where the decaying exponential function has been used as the creep kernel used for wood:

$$
K(t-\tau)=A_{1} \cdot e^{-\alpha_{1} \cdot(t-\tau)}
$$

where $A_{1}$ and $\alpha_{1}$ are constant coefficients found experimentally by the creep curves of the wooden elements under bending $[14,15]$ using the method of A.K. Malmeister $\left(A_{1}=0,0275\right.$, $\left.\alpha_{1}=0,0625\right)$.

Lets explain the coefficients $A_{1}$ and $\alpha_{1}$ included in the creep kernel:

$$
A_{1}=\frac{E_{l}-E_{w}(t)}{E_{w} \cdot \eta_{w}}
$$




$$
\alpha_{1}=\frac{E_{w}(t)}{E_{w} \cdot \eta_{w}}
$$

where $E_{w}$ и $E_{w}(t)$ are the instantaneous and long-lasting elastic modulus of wood, $\eta_{w}$ is the relaxation time coefficient, called the rheological constants, in this case, of wood and determined by the experimental creep curve (Fig. 1).

The instantaneous modulus of elasticity is deduced from the instantaneous strain at time $\mathrm{t}=0$, namely:

$$
E_{w}=\frac{\sigma}{\varepsilon_{0}}
$$

Durable elasticity modulus deduce from the formula:

$$
E_{w}(t)=\frac{\sigma}{\varepsilon_{(\infty)}}
$$

When processing experimental curves it is most difficult to determine $\varepsilon(\infty)$ due to the requirement of the very long load exposure of the sample. Therefore, it is advisable to determine the value of $\varepsilon(\infty)$ by an extrapolation method. Approximating the creep curve by the exponent:

$$
\varepsilon(t)=\frac{\sigma}{E_{w}(t)}\left(1-\frac{E_{w}-E_{w}(t)}{E_{l}} \cdot e^{\frac{t \cdot E_{w}(t)}{E_{w} \cdot \eta_{w}}}\right)
$$

you can derive a formula to determine the ultimate creep deformation by the extrapolation:

$$
\varepsilon(\infty)=\frac{\varepsilon_{1}^{2}-\varepsilon_{0} \cdot \varepsilon_{2}}{2 \cdot \varepsilon_{1}-\varepsilon_{0}-\varepsilon_{2}}
$$

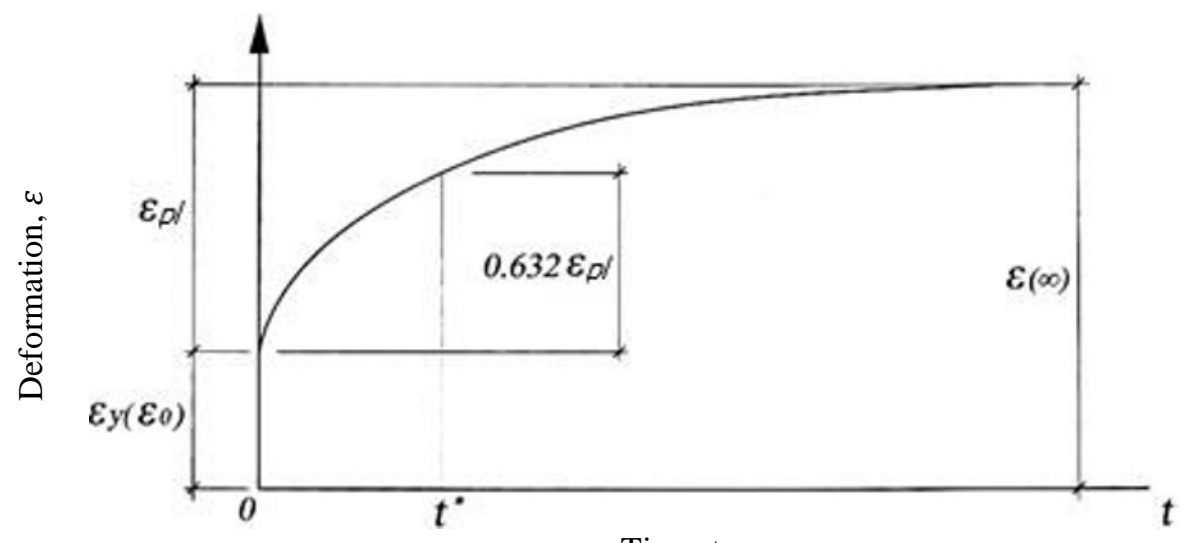

Time, $\mathrm{t}$

Fig. 1.. Experimental creep curve of wood.

Time $t_{1}$ and $t_{2}$ is chosen in such a way that the equation is valid

$$
t_{2}=2 \cdot t_{1}
$$


Deformations $\varepsilon_{0}, \varepsilon_{1}$ and $\varepsilon_{2}$ are determined by the experimental curve.

Sample left under the load over time:

$$
t^{*}=\frac{E_{w} \cdot \eta_{w}}{E_{w}(t)}
$$

will have the strain determined when $t=t^{*}$

$$
\begin{aligned}
\varepsilon\left(t^{*}\right)=\frac{\sigma}{E_{w}(t)}- & \left(\frac{\sigma}{E_{w}(t)}-\frac{\sigma}{E_{w}}\right) \cdot e^{-1}=\varepsilon_{y}+\varepsilon_{n}-\left(\varepsilon_{y}+\varepsilon_{n}+\varepsilon_{y}\right) \cdot e^{-1} \\
& =\varepsilon_{y}+\varepsilon_{n}\left(1-e^{-1}\right)=\varepsilon_{y}+0.632 \cdot \varepsilon_{n}
\end{aligned}
$$

Thus, the relaxation time coefficient is calculated using the formula:

$$
\eta_{w} \cdot t^{*}=\frac{E_{w}(t) \cdot t^{*}}{E_{w}}
$$

where the relaxation time $t^{*}-$ is determined from the experimental creep curve during strains (see Fig. 1).

The conducted experimental and theoretical investigations [16, 17] and the reinforced glued wooden structures observations during the operational process have shown that their stress-strain state changes significantly under the load over time. Glue compositions used to connect reinforcement with wood ensure their reliable joint operation, however, as the result of the wood creep where wood is the basic material, the redistribution of forces between the reinforcement and wood occurs there on and off. At the same time normal stresses in the reinforcement and the glue joint "reinforcement - wood" increase in the calculated cross sections while they decrease in the wood, which is caused by a change (decrease) in the wood elastic modulus [18]. In principle, this has a positive effect on the durability and reliability of the structures, as the reinforcement "unloads" the "weaker" material - wood over time, as a result of which the negative effect of the wood structure flaws on the load bearing capacity is reduced, which makes it possible to use the 3rd grade converted timber.

The effect of the period of loading must be considered when designing reinforced structures [19] since the neglect of the time factor can lead to the loss of the bearing capacity or to an unacceptable growth of strains (displacements).

The use of the formulas obtained for the practical calculation is difficult because of their bulkiness. It is known that in the area of the attenuated creep under the long-lasting static load, the structure acquires a stress-strain state after a certain time, which remains almost unchanged in the time following. In this regard, the mentioned formulas can be simplified by substituting variable operators for epy influence coefficients reflecting the steady stressstrain state [20-26].

Therefore, in connection with the redistribution of forces between the reinforcement and wood under the long-lasting constant load when calculating elements it is necessary to introduce the influence coefficient $K_{l}^{w}$ - taking into account the decrease in wood stresses over time and $K_{l}^{S}$ - taking into account the reinforcement increase in stresses over time. Then, when calculating the ultimate limit state, the normal stresses in the wood should be determined by the formula:

$$
\sigma_{l}(t)=\frac{M \cdot K_{l}^{w}}{W_{\text {red }}} \leq \frac{R_{b}}{\gamma_{n}}
$$


where $K_{l}^{w}$ - is a numerical value and is determined at t $\rightarrow \infty$ by the formula:

$$
K_{l}^{w}=\left[\frac{\alpha_{1}}{\beta_{1}}+\left(1-\frac{\alpha_{1}}{\beta_{1}}\right) \cdot e^{-\beta . t}\right]=\frac{\alpha_{1}}{\beta_{1}}
$$

and in the reinforcement by the formula:

$$
\sigma_{s}(t)=\frac{M \cdot n \cdot K_{l}^{s}}{W_{\text {red }}} \leq \frac{R_{s}}{\gamma_{n}}
$$

where $K_{l}^{s}-$ is the numerical value of the expression and is determined when $\mathrm{t} \rightarrow \infty$ by the formula:

$$
K_{l}^{S}=\left[\frac{A_{1}+\alpha_{1}}{\beta_{1}}+\left(1-\frac{A_{1}+\alpha_{1}}{\beta_{1}}\right) \cdot e^{-\beta . t}\right]=\frac{A_{1}+\alpha_{1}}{\beta_{1}}
$$

Stresses calculated from the formulas (13) and (15) correspond to steady-state longlasting.

Calculation of the serviceability limit states consists in finding the deflection of the reinforced element and comparing it with the specified value.

The steady-state deflection of the reinforced element [5] from the action of a uniformly distributed load is determined by the expression:

$$
f(t)=\left[\frac{5}{384} \cdot \frac{q \cdot l^{4}}{E_{l} \cdot I_{\text {red }}}+\frac{3 \cdot q \cdot l^{2}}{16 \cdot G_{l} \cdot F_{\text {red }}}\right] \cdot K_{l}^{s}
$$

For the rectangular beam with the width $b$ and the height $h$, having symmetrical reinforcement in tension and compression regions with area $\mathrm{A}$, we have:

- the moment of the wood inertia:

$$
I_{w}=\frac{b \cdot h^{3}}{12}
$$

- the moment of the reinforcement inertia:

$$
I_{s}=\frac{2 \cdot A \cdot h^{2}}{4}
$$

We find the ratio:

$$
\begin{gathered}
\frac{I_{s}}{I_{w}}=\frac{2 \cdot A \cdot h^{2} \cdot 12}{4 \cdot b \cdot h^{3}}=3 \cdot \mu \\
m_{1}=\frac{E_{s} \cdot I_{s}}{E_{w} \cdot I_{w}}=3 \cdot n \cdot \mu
\end{gathered}
$$

where $\mu=\frac{\sum A}{b \cdot h}$ - is the reinforcement coefficient;

$n=\frac{E_{S}}{E_{w}}-$ is the coefficient taking into account the ratio of the calculated elastic moduli of the reinforcement and wood.

Then the coefficient $\beta_{1}$ can be determined in this way: 


$$
\beta_{1}=\alpha_{1}+\frac{\mathrm{A}_{1} \cdot m_{1}}{1+m_{1}}=\alpha_{1}+\frac{\mathrm{A}_{1} \cdot 3 \cdot n \cdot \mu}{1+3 \cdot n \cdot \mu}
$$

Thus, for a symmetrically reinforced element of the rectangular section, expressions (15) and (17) can be written as:

$$
\begin{gathered}
K_{l}^{w}=\frac{\alpha_{1}}{\beta_{1}}+\frac{\alpha_{1}}{\alpha_{1}+\frac{\mathrm{A}_{1} \cdot 3 \cdot n \cdot \mu}{1+3 \cdot n \cdot \mu}} \leq 1 \\
K_{l}^{s}=\frac{A_{1}+\alpha_{1}}{\beta_{1}}+\frac{A_{1}+\alpha_{1}}{\alpha_{1}+\frac{\mathrm{A}_{1} \cdot 3 \cdot n \cdot \mu}{1+3 \cdot n \cdot \mu}} \geq 1
\end{gathered}
$$

Substituting the expressions (4) and (5) into (23) and (24) we get:

$$
\begin{gathered}
K_{l}^{w}=\frac{\alpha_{1}}{\alpha_{1}+\frac{\mathrm{A}_{1} \cdot 3 \cdot n \cdot \mu}{1+3 \cdot n \cdot \mu}}=\frac{E_{w}(t) \cdot(1+3 \cdot n \cdot \mu)}{E_{w}(t)+E_{w} \cdot 3 \cdot n \cdot \mu}=\frac{1+3 \cdot n \cdot \mu}{1+3 \cdot n \cdot \mu \cdot m_{l}} \leq 1 \\
K_{l}^{s}=\frac{A_{1}+\alpha_{1}}{\alpha_{1}+\frac{\mathrm{A}_{1} \cdot 3 \cdot n \cdot \mu}{1+3 \cdot n \cdot \mu}}+\frac{E_{w} \cdot(1+3 \cdot n \cdot \mu)}{E_{w}(t)+E_{w} \cdot 3 \cdot n \cdot \mu}+\frac{1+3 \cdot n \cdot \mu}{m_{w}+3 \cdot n \cdot \mu} \geq 1
\end{gathered}
$$

где

$$
m_{l}=\frac{E_{w}(t)}{E_{w}}
$$

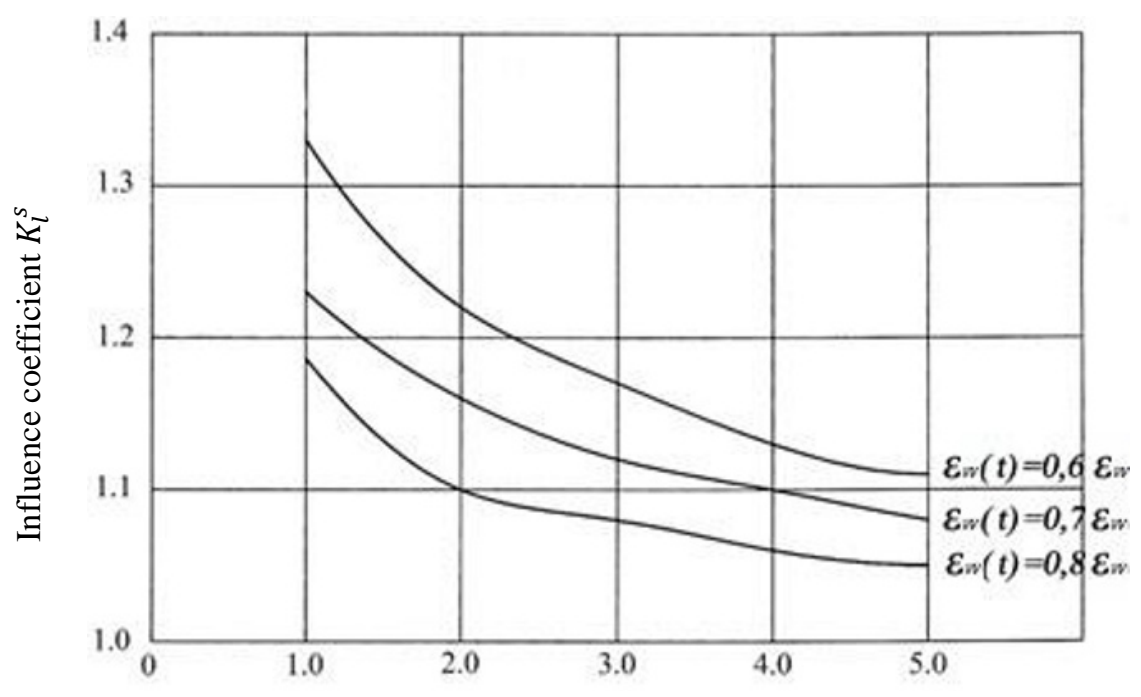

The coefficient of the reinforcement, $\%$

Fig. 2. Dependence of the redistribution of forces coefficients in the reinforcement on the reinforcement coefficients 


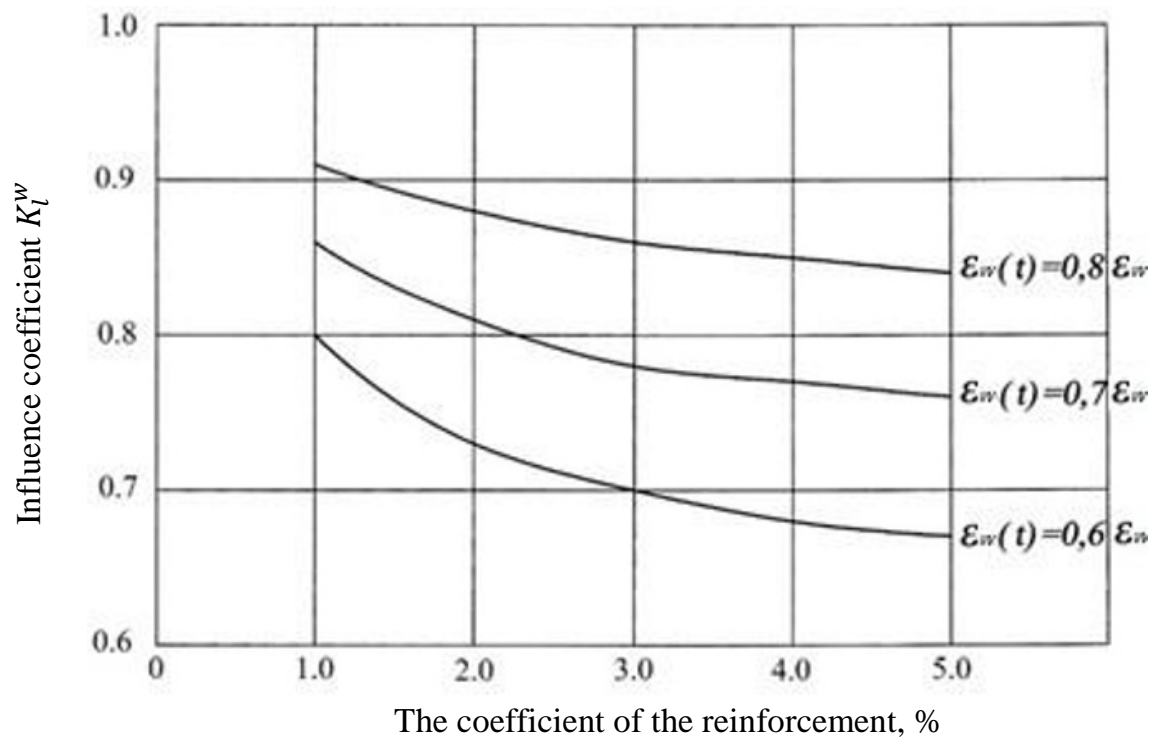

Fig. 3. Dependence of the redistribution of forces coefficients in wood on the reinforcement coefficients

The obtained expressions (26) and (27) show that the value of the coefficients $K_{l}^{w}$ and $K_{l}^{S}$ depend on the reinforcement coefficient of the cross section of the wood and reinforcement elastic characteristics (Fig. 2 and 3).

The coefficient $K_{l}^{w}$ takes into account the reduction of stresses in the wood, and $K_{l}^{S}$ - the increase of the stresses in the reinforcement under the bending of the reinforced elements by the long-lasting load.

The above formulas describe the work of the reinforced wood bending elements in the damped creep area. When static loads exceed the limit of the long lasting bearing capacity the deformations increase in time inevitably ends in the structural failure.

\section{Conclusions}

The method based on the theory of elastic heredity can be used to investigate the stress-strain state of the reinforced wooden structures.

Changeability of the wood elasticity modulus over time has a significant effect on the redistribution of stresses between the wood and reinforcement. If the instantaneous elasticity modulus is assumed to be a constant value in calculations, then this, as a rule, implies an increase in the factor of assurance.

Allowance for the wood creep in the design of the reinforced wooden structures will allow to determine the stress - strain state of the structure in the design more precisely. Therefore, when developing the reinforced wooden structures design standards, this factor must be taken into account. 


\section{References}

1. F.J. Rescalvo, E. Suarez, I. Valverde-Palacios, J.M. Santiago-Zaragoza, A. Gallego, Composite Structures, 206 (2018)

2. R. Harris, Wood Composites, 8 (2015)

3. M. Lyu, X. Zhu, Q. Yang, Engineering Structures, 151 (2017)

4. T. Zmijewki, D. Wojtowicz-Jankowska, IOP Conference Series: Materials Science and Engineering, 245(8) (2017)

5. M. Corradi, A. Borri, L. Righetti, E. Speranzini, Composites Part B: Engineering, 113 (2017)

6. B. D'Amico, A. Kermani, J. Porteous, R. Dhonju, B. Zhang, Construction and Building Materials, 129 (2016)

7. G. Schiro, I. Giongo, W. Sebastian, D. Riccadonna, M. Piazza, Construction and Building Materials, 171 (2018)

8. D.A. García, R. Sampaio, M.B. Rosales, Journal of the Brazilian Society of Mechanical Sciences and Engineering, 38(8) (2016)

9. L.-M. Ottenhaus, M. Li, T. Smith, Engineering Structures, 176 (2018)

10. P. Dietsch, Construction and Building Materials, 150 (2017)

11. R. Sardiko, K. Rocens, J. Iejavs, V. Jakovlevs, K. Ziverts, IOP Conference Series: Materials Science and Engineering, 251(1) (2017)

12. L. Franzoni, A. Lebée, F. Lyon, G. Forêt, Engineering Structures, 141 (2017)

13. C.B. Ong, Wood Composites, 7 (2015)

14. L.A. Basterra, J.A. Balmori, L. Morillas, L. Acuña, M. Casado, Construction and Building Materials, 154 (2017)

15. A. Lokaj, K. Vavrusova, D. Mikolasek, Procedia Engineering, 190 (2017)

16. N. Keipour, H.R. Valipour, M.A. Bradford, Engineering Structures, 174 (2018)

17. F. Solarino, L. Giresini, W.-S. Chang, H. Huang, Buildings, 7(4) (2017)

18. G. Rinaldin, M. Fragiacomo, C. Amadio, Soil Dynamics and Earthquake Engineering, 100 (2017)

19. K. Schober, A. Harte, R. Kliger, R. Jockwer, Q. Xu, J. Chen, Construction and Building Materials, 97 (2015)

20. A.S. Rassokhin, A.N. Ponomarev, O.L. Figovsky, Magazine of Civil Engineering, 79(3), 132-139 (2018)

21. I.K. Rasha, O.N. Pertseva, A.Yu. Lazareva, G.V. Martynov, Mag. Civ. Eng., 69(1) (2017)

22. A. Chulkova, S. Lukichev, M. Romanovich, MATEC Web Conf., 86, 02019 (2016)

23. A.N. Ponomarev, A.S. Rassokhin, Mag. Civ. Eng., 68 (8) (2016)

24. T. Saknite, D. Serdjuks, V. Goremikins, L. Pakrastins, N.I. Vatin, Mag. Civ. Eng., 64(4) (2016)

25. I.K. Yaichnikov, V.P. Suschenko, Teoriya i Praktika Fizicheskoy Kultury (2014)

26. S. Franke, B. Franke, A. Harte, Construction and Building Materials, 97 (2015) 